\title{
Rational Solutions of the Sasano System of Type $A_{5}^{(2)}$
}

Kazuhide MATSUDA

Department of Engineering Science, Niihama National College of Technology,

7-1 Yagumo-chou, Niihama, Ehime, 792-8580, Japan

E-mail: matsuda@sci.niihama-nct.ac.jp

Received November 5, 2010, in final form March 17, 2011; Published online March 25, 2011

doi:10.3842/SIGMA.2011.030

\begin{abstract}
In this paper, we completely classify the rational solutions of the Sasano system of type $A_{5}^{(2)}$, which is given by the coupled Painlevé III system. This system of differential equations has the affine Weyl group symmetry of type $A_{5}^{(2)}$.
\end{abstract}

Key words: affine Weyl group; rational solutions; Sasano system

2010 Mathematics Subject Classification: 33E17; 34M55

\section{Introduction}

Paul Painlevé and his colleagues [21, 4] intended to find new transcendental functions defined by second order nonlinear differential equations. In general, nonlinear differential equations have moving branch points. If a solution has moving branch points, it is too complicated and is not worth considering. Therefore, they determined the second order nonlinear differential equations with rational coefficients which have no moving branch points. As a result, the standard forms of such equations turned out to be given by the following six equations:

$$
\begin{aligned}
& P_{\mathrm{I}}: \quad y^{\prime \prime}=6 y^{2}+t, \\
& P_{\mathrm{II}}: \quad y^{\prime \prime}=2 y^{3}+t y+\alpha, \\
& P_{\mathrm{III}}: \quad y^{\prime \prime}=\frac{1}{y}\left(y^{\prime}\right)^{2}-\frac{1}{t} y^{\prime}+\frac{1}{t}\left(\alpha y^{2}+\beta\right)+\gamma y^{3}+\frac{\delta}{y}, \\
& P_{\mathrm{IV}}: \quad y^{\prime \prime}=\frac{1}{2 y}\left(y^{\prime}\right)^{2}+\frac{3}{2} y^{3}+4 t y^{2}+2\left(t^{2}-\alpha\right) y+\frac{\beta}{y}, \\
& P_{\mathrm{V}}: \quad y^{\prime \prime}=\left(\frac{1}{2 y}+\frac{1}{y-1}\right)\left(y^{\prime}\right)^{2}-\frac{1}{t} y^{\prime}+\frac{(y-1)^{2}}{t^{2}}\left(\alpha y+\frac{\beta}{y}\right)+\frac{\gamma}{t} y+\delta \frac{y(y+1)}{y-1}, \\
& P_{\mathrm{VI}}: \quad y^{\prime \prime}=\frac{1}{2}\left(\frac{1}{y}+\frac{1}{y-1}+\frac{1}{y-t}\right)\left(y^{\prime}\right)^{2}-\left(\frac{1}{t}+\frac{1}{t-1}+\frac{1}{y-t}\right) y^{\prime} \\
& +\frac{y(y-1)(y-t)}{t^{2}(t-1)^{2}}\left(\alpha+\beta \frac{t}{y^{2}}+\gamma \frac{t-1}{(y-1)^{2}}+\delta \frac{t(t-1)}{(y-t)^{2}}\right),
\end{aligned}
$$

where $^{\prime}=d / d t$ and $\alpha, \beta, \gamma, \delta$ are all complex parameters. In this article, our concern is with the Bäcklund transformations and special solutions which are given by rational, algebraic functions or classical special functions.

Each of $P_{J}(J=$ II, III, IV, V, VI) has Bäcklund transformations, which transform solutions into other solutions of the same equation with different parameters. It was shown by Okamoto $[17,18,19,20]$ that the Bäcklund transformation groups of the Painlevé equations except for $P_{\mathrm{I}}$ are isomorphic to the extended affine Weyl groups. For $P_{\mathrm{II}}, P_{\mathrm{III}}, P_{\mathrm{IV}}, P_{\mathrm{V}}$, and $P_{\mathrm{VI}}$, the Bäcklund transformation groups correspond to $A_{1}^{(1)}, A_{1}^{(1)} \bigoplus A_{1}^{(1)}, A_{2}^{(1)}, A_{3}^{(3)}$, and $D_{4}^{(1)}$, respectively. 
While generic solutions of the Painlevé equations are "new transcendental functions", there are special solutions which are expressible in terms of rational, algebraic, or classical special functions.

For example, Airault [1] constructed explicit rational solutions of $P_{\mathrm{II}}$ and $P_{\mathrm{IV}}$ with their Bäcklund transformations. Milne, Clarkson and Bassom [13] treated $P_{\mathrm{III}}$, and described their Bäcklund transformations and exact solution hierarchies, which are given by rational, algebraic, or certain Bessel functions. Bassom, Clarkson and Hicks [2] dealt with $P_{\mathrm{IV}}$, and described their Bäcklund transformations and exact solution hierarchies, which are expressed by rational functions, the parabolic cylinder functions or the complementary error functions. Clarkson [3] studied some rational and algebraic solutions of $P_{\text {III }}$ and showed that these solutions are expressible in terms of special polynomials defined by second order, bilinear differential-difference equations which are equivalent to Toda equations.

Furthermore, the rational solutions of $P_{J}(J=$ II, III, IV, V, VI) were classified by Yablonski and Vorobev [25, 24], Gromak [6, 5], Murata [14, 15], Kitaev, Law and McLeod [8], Mazzoco [11] and Yuang and Li [26].

Noumi and Yamada [16] discovered the equation of type $A_{l}^{(1)}(l \geq 2)$, whose Bäcklund transformation group is isomorphic to the extended affine Weyl group $\tilde{W}\left(A_{l}^{(1)}\right)$. The Noumi and Yamada systems of types $A_{2}^{(1)}$ and $A_{3}^{(1)}$ correspond to the fourth and fifth Painlevé equations, respectively. Moreover, we $[9,10]$ classified the rational solutions of the Noumi and Yamada systems of types $A_{4}^{(1)}$ and $A_{5}^{(1)}$.

Sasano [22] found the coupled Painlevé V and VI systems which have the affine Weyl group symmetries of types $D_{5}^{(1)}$ and $D_{6}^{(1)}$. In addition, he [23] obtained the equation of the affine Weyl group symmetry of type $A_{5}^{(2)}$, which is defined by

$$
A_{5}^{(2)}\left(\alpha_{j}\right)_{0 \leq j \leq 3}\left\{\begin{array}{l}
t q_{1}^{\prime}=2 q_{1}^{2} p_{1}-q_{1}^{2}+\left(\alpha_{0}+\alpha_{1}+\alpha_{3}\right) q_{1}-t+4 t p_{2}+2 q_{1} q_{2} p_{2}, \\
t p_{1}^{\prime}=-2 q_{1} p_{1}^{2}+2 q_{1} p_{1}-\left(\alpha_{0}+\alpha_{1}+\alpha_{3}\right) p_{1}+\alpha_{0}-2 p_{1} q_{2} p_{2} \\
t q_{2}^{\prime}=2 q_{2}^{2} p_{2}-q_{2}^{2}+\left(\alpha_{0}+\alpha_{1}+\alpha_{3}\right) q_{2}-t+4 t p_{1}+2 q_{1} p_{1} q_{2} \\
t p_{2}^{\prime}=-2 q_{2} p_{2}^{2}+2 q_{2} p_{2}-\left(\alpha_{0}+\alpha_{1}+\alpha_{3}\right) p_{2}+\alpha_{1}-2 q_{1} p_{1} p_{2} \\
\alpha_{0}+\alpha_{1}+2 \alpha_{2}+\alpha_{3}=1 / 2
\end{array}\right.
$$

where $^{\prime}=d / d t$. This system of differential equations is also expressed by the Hamiltonian system:

$$
t \frac{d q_{1}}{d t}=\frac{\partial H}{\partial p_{1}}, \quad t \frac{d p_{1}}{d t}=-\frac{\partial H}{\partial q_{1}}, \quad t \frac{d q_{2}}{d t}=\frac{\partial H}{\partial p_{2}}, \quad t \frac{d p_{2}}{d t}=-\frac{\partial H}{\partial q_{2}}
$$

where the Hamiltonian $H$ is given by

$$
\begin{aligned}
H= & q_{1}^{2} p_{1}^{2}-q_{1}^{2} p_{1}+\left(\alpha_{0}+\alpha_{1}+\alpha_{3}\right) q_{1} p_{1}-\alpha_{0} q_{1}-t p_{1} \\
& +q_{2}^{2} p_{2}^{2}-q_{2}^{2} p_{2}+\left(\alpha_{0}+\alpha_{1}+\alpha_{3}\right) q_{2} p_{2}-\alpha_{1} q_{2}-t p_{2}+4 t p_{1} p_{2}+2 q_{1} p_{1} q_{2} p_{2} .
\end{aligned}
$$

Let us note that Mazzocco and Mo [12] studied the Hamiltonian structure of the $P_{\mathrm{II}}$ hierarchy, and Hone [7] studied the coupled Painleve systems from the similarity reduction of the Hirota-Satsuma system and another gauge-related system, and presented their Bäcklund transformations and special solutions.

$A_{5}^{(2)}\left(\alpha_{j}\right)_{0 \leq j \leq 3}$ has the Bäcklund transformations $s_{0}, s_{1}, s_{2}, s_{3}, \pi$, which are given by

$$
s_{0}: \quad(*) \rightarrow\left(q_{1}+\frac{\alpha_{0}}{p_{1}}, p_{1}, q_{2}, p_{2}, t ;-\alpha_{0}, \alpha_{1}, \alpha_{2}+\alpha_{0}, \alpha_{3}\right),
$$




$$
\begin{array}{ll}
s_{1}: & (*) \rightarrow\left(q_{1}, p_{1}, q_{2}+\frac{\alpha_{1}}{p_{2}}, p_{2}, t ; \alpha_{0},-\alpha_{1}, \alpha_{2}+\alpha_{1}, \alpha_{3}\right), \\
s_{2}: & (*) \rightarrow\left(q_{1}, p_{1}-\frac{\alpha_{2} q_{2}}{q_{1} q_{2}+t}, q_{2}, p_{2}+\frac{\alpha_{2} q_{1}}{q_{1} q_{2}+t}, t ; \alpha_{0}+\alpha_{2}, \alpha_{1}+\alpha_{2},-\alpha_{2}, \alpha_{3}+2 \alpha_{2}\right), \\
s_{3}: & (*) \rightarrow\left(q_{1}+\frac{\alpha_{3}}{p_{1}+p_{2}-1}, p_{1}, q_{2}+\frac{\alpha_{3}}{p_{1}+p_{2}-1}, p_{2}, t ; \alpha_{0}, \alpha_{1}, \alpha_{2}+\alpha_{3},-\alpha_{3}\right), \\
\pi: & (*) \rightarrow\left(q_{2}, p_{2}, q_{1}, p_{1}, t ; \alpha_{1}, \alpha_{0}, \alpha_{2}, \alpha_{3}\right),
\end{array}
$$

with the notation $(*)=\left(q_{1}, p_{1}, q_{2}, p_{2}, t ; \alpha_{0}, \alpha_{1}, \alpha_{2}, \alpha_{3}\right)$. The Bäcklund transformation group $\left\langle s_{0}, s_{1}, s_{2}, s_{3}, \pi\right\rangle$ is isomorphic to the affine Weyl group of type $A_{5}^{(2)}$.

Our main theorem is as follows:

Theorem 1.1. For a rational solution of $A_{5}^{(2)}\left(\alpha_{j}\right)_{0 \leq j \leq 3}$, by some Bäcklund transformations, the solution and parameters can be transformed so that

$$
\begin{aligned}
& \left(q_{1}, p_{1}, q_{2}, p_{2}\right)=(0,1 / 4,0,1 / 4) \quad \text { and } \\
& \left(\alpha_{0}, \alpha_{1}, \alpha_{2}, \alpha_{3}\right)=\left(\alpha_{3} / 2, \alpha_{3} / 2, \alpha_{2}, \alpha_{3}\right)=\left(\alpha_{3} / 2, \alpha_{3} / 2,1 / 4-\alpha_{3}, \alpha_{3}\right),
\end{aligned}
$$

respectively. Furthermore, for $A_{5}^{(2)}\left(\alpha_{j}\right)_{0 \leq j \leq 3}$, there exists a rational solution if and only if one of the following occurs:

$$
\begin{array}{rrr}
(1) & -2 \alpha_{0}+\alpha_{3} \in \mathbb{Z}, & -2 \alpha_{1}+\alpha_{3} \in \mathbb{Z}, \\
(2) & -2 \alpha_{0}+\alpha_{3} \in \mathbb{Z}, & 2 \alpha_{1}+\alpha_{3} \in \mathbb{Z}, \\
(3) & 2 \alpha_{0}+\alpha_{3} \in \mathbb{Z}, & -2 \alpha_{1}+\alpha_{3} \in \mathbb{Z}, \\
(4) & 2 \alpha_{0}+\alpha_{3} \in \mathbb{Z}, & 2 \alpha_{1}+\alpha_{3} \in \mathbb{Z}, \\
(5) & -2 \alpha_{0}+\alpha_{3} \in \mathbb{Z}, & \alpha_{3}-1 / 2 \in \mathbb{Z}, \\
(6) & -2 \alpha_{1}+\alpha_{3} \in \mathbb{Z}, & \alpha_{3}-1 / 2 \in \mathbb{Z} .
\end{array}
$$

This paper is organized as follows. In Section 2 , for $A_{5}^{(2)}\left(\alpha_{j}\right)_{0 \leq j \leq 3}$, we determine meromorphic solutions at $t=\infty$. Then, we find that the constant terms $a_{\infty, 0}, c_{\infty, 0}$ of the Laurent series of $q_{1}, q_{2}$ at $t=\infty$ are given by

$$
a_{\infty, 0}:=-2 \alpha_{0}+\alpha_{3}, \quad c_{\infty, 0}:=-2 \alpha_{1}+\alpha_{3},
$$

respectively.

In Section 3 , for $A_{5}^{(2)}\left(\alpha_{j}\right)_{0 \leq j \leq 3}$, we determine meromorphic solutions at $t=0$. Then, we see that the constant terms $a_{0,0}, c_{0,0}$ of the Laurent series of $q_{1}, q_{2}$ at $t=0$ are given by the parameters $\alpha_{0}, \alpha_{1}, \alpha_{2}, \alpha_{3}$.

In Section 4 , for $A_{5}^{(2)}\left(\alpha_{j}\right)_{0 \leq j \leq 3}$, we treat meromorphic solutions at $t=c \in \mathbb{C}^{*}$, where in this paper, $\mathbb{C}^{*}$ means the set of nonzero complex numbers. Then, we observe that $q_{1}, q_{2}$ have both a pole of order of at most one at $t=c$ and the residues of $q_{1}, q_{2}$ at $t=c$ are expressed by $n c$ $(n \in \mathbb{Z})$. Thus, it follows that

$$
a_{\infty, 0}-a_{0,0} \in \mathbb{Z}, \quad c_{\infty, 0}-c_{0,0} \in \mathbb{Z},
$$

which gives a necessary condition for $A_{5}^{(2)}\left(\alpha_{j}\right)_{0 \leq j \leq 3}$ to have a rational solution.

In Section 5, using the meromorphic solution at $t=\infty, 0$, we first compute the constant terms of the Laurent series of the Hamiltonian at $t=\infty, 0$. Furthermore, by the meromorphic solution at $=c \in \mathbb{C}^{*}$, we calculate the residue of $H$ at $t=c$. 
In Section 6, by equation (1.1), we obtain the necessary conditions for $A_{5}^{(2)}\left(\alpha_{j}\right)_{0 \leq j \leq 3}$ to have rational solutions, which are given in our main theorem. Furthermore, we show that if there exists a rational solution for $A_{5}^{(2)}\left(\alpha_{j}\right)_{0 \leq j \leq 3}$, the parameters can be transformed so that $-2 \alpha_{0}+\alpha_{3} \in \mathbb{Z},-2 \alpha_{1}+\alpha_{3} \in \mathbb{Z}$.

In Section 7, we define shift operators, and for a rational solution of $A_{5}^{(2)}\left(\alpha_{j}\right)_{0 \leq j \leq 3}$, we transform the parameters to

$$
\left(\alpha_{0}, \alpha_{1}, \alpha_{2}, \alpha_{3}\right)=\left(\alpha_{3} / 2, \alpha_{3} / 2, \alpha_{2}, \alpha_{3}\right) \text {. }
$$

In Section 8, we determine rational solutions of $A_{5}^{(2)}\left(\alpha_{3} / 2, \alpha_{3} / 2, \alpha_{2}, \alpha_{3}\right)$ and prove our main theorem.

In Appendix A, using the shift operators, we give examples of rational solutions.

\section{Meromorphic solutions at $t=\infty$}

In this section, for $A_{5}^{(2)}\left(\alpha_{j}\right)_{0 \leq j \leq 3}$, we treat meromorphic solutions at $t=\infty$. For the purpose, in this paper, we define the coefficients of the Laurent series of $q_{1}, p_{1}, q_{2}, p_{2}$ at $t=\infty$ by $a_{\infty, k}$, $b_{\infty, k}, c_{\infty, k}, d_{\infty, k}, k \in \mathbb{Z}$.

\subsection{The case where $q_{1}, p_{1}, q_{2}, p_{2}$ are all holomorphic at $t=\infty$}

Proposition 2.1. Suppose that for $A_{5}^{(2)}\left(\alpha_{j}\right)_{0 \leq j \leq 3}$, there exists a solution such that $q_{1}, p_{1}, q_{2}$, $p_{2}$ are all holomorphic at $t=\infty$. Then,

$$
\left\{\begin{array}{l}
q_{1}=\left(-2 \alpha_{0}+\alpha_{3}\right)+\cdots, \\
p_{1}=1 / 4+\left(-2 \alpha_{1}+\alpha_{3}\right)\left(-2 \alpha_{1}-\alpha_{3}\right) t^{-1} / 4+\cdots, \\
q_{2}=\left(-2 \alpha_{1}+\alpha_{3}\right)+\cdots, \\
p_{2}=1 / 4+\left(-2 \alpha_{0}+\alpha_{3}\right)\left(-2 \alpha_{0}-\alpha_{3}\right) t^{-1} / 4+\cdots .
\end{array}\right.
$$

Proposition 2.2. Suppose that for $A_{5}^{(2)}\left(\alpha_{j}\right)_{0 \leq j \leq 3}$, there exists a solution such that $q_{1}, p_{1}, q_{2}$, $p_{2}$ are all holomorphic at $t=\infty$. Then, it is unique.

Proof. We set

$$
\begin{aligned}
& q_{1}=a_{\infty, 0}+a_{\infty,-1} t^{-1}+\cdots+a_{\infty,-(k-1)} t^{-(k-1)}+a_{\infty,-k} t^{-k}+a_{\infty,-(k+1)} t^{-(k+1)}+\cdots, \\
& p_{1}=1 / 4+b_{\infty,-1} t^{-1}+\cdots+b_{\infty,-(k-1)} t^{-(k-1)}+b_{\infty,-k} t^{-k}+b_{\infty,-(k+1)} t^{-(k+1)}+\cdots, \\
& q_{2}=c_{\infty, 0}+c_{\infty,-1} t^{-1}+\cdots+c_{\infty,-(k-1)} t^{-(k-1)}+c_{\infty,-k} t^{-k}+c_{\infty,-(k+1)} t^{-(k+1)}+\cdots, \\
& p_{2}=1 / 4+d_{\infty,-1} t^{-1}+\cdots+d_{\infty,-(k-1)} t^{-(k-1)}+d_{\infty,-k} t^{-k}+d_{\infty,-(k+1)} t^{-(k+1)}+\cdots,
\end{aligned}
$$

where $a_{\infty, 0}, b_{\infty,-1}, c_{\infty, 0}, d_{\infty,-1}$ all have been determined.

Comparing the coefficients of the terms $t^{-k}(k \geq 1)$ in

$$
\begin{aligned}
& t p_{1}^{\prime}=-2 q_{1} p_{1}^{2}+2 q_{1} p_{1}-\left(\alpha_{0}+\alpha_{1}+\alpha_{3}\right) p_{1}+\alpha_{0}-2 p_{1} q_{2} p_{2}, \\
& t p_{2}^{\prime}=-2 q_{2} p_{2}^{2}+2 q_{2} p_{2}-\left(\alpha_{0}+\alpha_{1}+\alpha_{3}\right) p_{2}+\alpha_{1}-2 q_{1} p_{1} p_{2},
\end{aligned}
$$

we have

$$
\begin{aligned}
& 3 a_{\infty,-k} / 8-c_{\infty,-k} / 8=-k b_{\infty,-k}+\left(\alpha_{0}+\alpha_{1}+\alpha_{3}\right) b_{\infty,-k} \\
& \quad+2 \sum a_{\infty,-l} b_{\infty,-m} b_{\infty,-n}-2 \sum a_{\infty,-l} b_{\infty,-m}+2 \sum c_{\infty,-l} b_{\infty,-m} d_{\infty,-n},
\end{aligned}
$$




$$
\begin{aligned}
& -a_{\infty,-k} / 8+3 c_{\infty,-k} / 8=-k d_{\infty,-k}+\left(\alpha_{0}+\alpha_{1}+\alpha_{3}\right) d_{\infty,-k} \\
& \quad+2 \sum c_{\infty,-l} d_{\infty,-m} d_{\infty,-n}-2 \sum c_{\infty,-l} d_{\infty,-m}+2 \sum a_{\infty,-l} b_{\infty,-m} d_{\infty,-n},
\end{aligned}
$$

where the first and third sums extend over nonnegative integers $l, m, n$ such that $l+m+n=k$ and $0 \leq l<k$, and the second sums extend over nonnegative integers $l, m$ such that $l+m=k$ and $m \geq 1$. Therefore, $a_{\infty,-k}, c_{\infty,-k}$ are both inductively determined.

Comparing the coefficients of the terms $t^{-k}(k \geq 1)$ in

$$
\begin{aligned}
& t q_{1}^{\prime}=2 q_{1}^{2} p_{1}-q_{1}^{2}+\left(\alpha_{0}+\alpha_{1}+\alpha_{3}\right) q_{1}-t+4 t p_{2}+2 q_{1} q_{2} p_{2} \\
& t q_{2}^{\prime}=2 q_{2}^{2} p_{2}-q_{2}^{2}+\left(\alpha_{0}+\alpha_{1}+\alpha_{3}\right) q_{2}-t+4 t p_{1}+2 q_{1} p_{1} q_{2}
\end{aligned}
$$

we obtain

$$
\begin{aligned}
& 4 d_{\infty,-(k+1)}=-k a_{\infty,-k}-\left(\alpha_{0}+\alpha_{1}+\alpha_{3}\right) a_{\infty,-k} \\
& \quad-2 \sum a_{\infty,-l} a_{\infty,-m} b_{\infty,-n}+\sum a_{\infty,-l} a_{\infty,-m}-2 \sum a_{\infty,-l} c_{\infty,-m} d_{\infty,-n}, \\
& 4 b_{\infty,-(k+1)}=-k c_{\infty,-k}-\left(\alpha_{0}+\alpha_{1}+\alpha_{3}\right) c_{\infty,-k} \\
& \quad-2 \sum c_{\infty,-l} c_{\infty,-m} d_{\infty,-n}+\sum c_{\infty,-l} c_{\infty,-m}-2 \sum c_{\infty,-l} a_{\infty,-m} b_{\infty,-n},
\end{aligned}
$$

where the first and third sums extend over nonnegative integers $l, m, n$ such that $l+m+n=k$, and the second sums extend over nonnegative integers $l, m$ such that $l+m=k$. Therefore, $b_{\infty,-(k+1)}, d_{\infty,-(k+1)}$ are both inductively determined, which proves the proposition.

\subsection{The case where one of $\left(q_{1}, p_{1}, q_{2}, p_{2}\right)$ has a pole at $t=\infty$}

In this subsection, we deal with the case in which one of $\left(q_{1}, p_{1}, q_{2}, p_{2}\right)$ has a pole at $t=\infty$. For the purpose, by $\pi$, we have only to consider the following two cases:

(1) $q_{1}$ has a pole at $t=\infty$ and $p_{1}, q_{2}, p_{2}$ are all holomorphic at $t=\infty$,

(2) $p_{1}$ has a pole at $t=\infty$ and $q_{1}, q_{2}, p_{2}$ are all holomorphic at $t=\infty$.

\subsubsection{The case where $q_{1}$ has a pole at $t=\infty$}

Proposition 2.3. For $A_{5}^{(2)}\left(\alpha_{j}\right)_{0 \leq j \leq 3}$, there exists no solution such that $q_{1}$ has a pole at $t=\infty$ and $p_{1}, q_{2}, p_{2}$ are all holomorphic at $t=\infty$.

\subsubsection{The case where $p_{1}$ has a pole at $t=\infty$}

Proposition 2.4. For $A_{5}^{(2)}\left(\alpha_{j}\right)_{0 \leq j \leq 3}$, there exists no solution such that $p_{1}$ has a pole at $t=\infty$ and $q_{1}, q_{2}, p_{2}$ are all holomorphic at $t=\infty$.

\subsection{The case where two of $\left(q_{1}, p_{1}, q_{2}, p_{2}\right)$ have a pole at $t=\infty$}

In this subsection, we deal with the case in which two of $\left(q_{1}, p_{1}, q_{2}, p_{2}\right)$ has a pole at $t=\infty$. For the purpose, by $\pi$, we have only to consider the following four cases:

(1) $q_{1}, p_{1}$ have both a pole at $t=\infty$ and $q_{2}, p_{2}$ are both holomorphic at $t=\infty$,

(2) $q_{1}, q_{2}$ have both a pole at $t=\infty$ and $p_{1}, p_{2}$ are both holomorphic at $t=\infty$,

(3) $q_{1}, p_{2}$ have both a pole at $t=\infty$ and $p_{1}, q_{2}$ are both holomorphic at $t=\infty$,

(4) $p_{1}, p_{2}$ have both a pole at $t=\infty$ and $q_{1}, q_{2}$ are both holomorphic at $t=\infty$. 


\subsubsection{The case where $q_{1}, p_{1}$ have a pole at $t=\infty$}

Proposition 2.5. For $A_{5}^{(2)}\left(\alpha_{j}\right)_{0 \leq j \leq 3}$, there exists no solution such that $q_{1}, p_{1}$ have both a pole at $t=\infty$ and $q_{2}, p_{2}$ are both holomorphic at $t=\infty$.

\subsubsection{The case where $q_{1}, q_{2}$ have a pole at $t=\infty$}

Proposition 2.6. For $A_{5}^{(2)}\left(\alpha_{j}\right)_{0 \leq j \leq 3}$, there exists no solution such that $q_{1}$, $q_{2}$ have both a pole at $t=\infty$ and $p_{1}, p_{2}$ are both holomorphic at $t=\infty$.

\subsubsection{The case where $q_{1}, p_{2}$ have a pole at $t=\infty$}

By direct calculation, we can obtain the following two lemmas:

Lemma 2.1. Suppose that for $A_{5}^{(2)}\left(\alpha_{j}\right)_{0 \leq j \leq 3}, q_{1} \equiv 0$. Then, one of the following occurs:
(1) $\alpha_{0}=\frac{1}{4}, \quad \alpha_{3}=\frac{1}{2}, \quad$ and
$\left(q_{1}, p_{1}, q_{2}, p_{2}\right)=\left(0, \frac{1}{4}+\frac{\left(4 \alpha_{1}-1\right)\left(4 \alpha_{1}+1\right)}{16 t},-2 \alpha_{1}+\frac{1}{2}, \frac{1}{4}\right)$,
(2) $\quad \alpha_{0}=\frac{\alpha_{3}}{2}, \quad \alpha_{1}=\frac{\alpha_{3}}{2}, \quad$ and $\quad\left(q_{1}, p_{1}, q_{2}, p_{2}\right)=\left(0, \frac{1}{4}, 0, \frac{1}{4}\right)$,
(3) $\quad \alpha_{0}=\frac{\alpha_{3}}{2}, \quad \alpha_{1}=-\frac{\alpha_{3}}{2}, \quad$ and $\quad\left(q_{1}, p_{1}, q_{2}, p_{2}\right)=\left(0, \frac{1}{4}, 2 \alpha_{3}, \frac{1}{4}\right)$.

Lemma 2.2. Suppose that for $A_{5}^{(2)}\left(\alpha_{j}\right)_{0 \leq j \leq 3}, q_{2} \equiv 0$. Then, one of the following occurs:
(1) $\quad \alpha_{1}=\frac{1}{4}, \quad \alpha_{3}=\frac{1}{2}, \quad$ and $\left(q_{1}, p_{1}, q_{2}, p_{2}\right)=\left(-2 \alpha_{0}+\frac{1}{2}, \frac{1}{4}, 0, \frac{1}{4}+\frac{\left(4 \alpha_{0}-1\right)\left(4 \alpha_{0}+1\right)}{16 t}\right)$,
(2) $\quad \alpha_{0}=\frac{\alpha_{3}}{2}, \quad \alpha_{1}=\frac{\alpha_{3}}{2}, \quad$ and $\quad\left(q_{1}, p_{1}, q_{2}, p_{2}\right)=\left(0, \frac{1}{4}, 0, \frac{1}{4}\right)$,
(3) $\alpha_{0}=-\frac{\alpha_{3}}{2}, \quad \alpha_{1}=\frac{\alpha_{3}}{2}, \quad$ and $\quad\left(q_{1}, p_{1}, q_{2}, p_{2}\right)=\left(2 \alpha_{3}, \frac{1}{4}, 0, \frac{1}{4}\right)$.

By Lemma 2.2, we find that $q_{2} \not \equiv 0$. Now, let us assume that $q_{1}$ has a pole of order $n_{0}$ $\left(n_{0} \geq 1\right)$ and $p_{2}$ has a pole of order $n_{3}\left(n_{3} \geq 1\right)$.

Lemma 2.3. For $A_{5}^{(2)}\left(\alpha_{j}\right)_{0 \leq j \leq 3}$, there exists a solution such that $q_{1}$, $p_{2}$ have both a pole at $t=\infty$ and $p_{1}, q_{2}$ are both holomorphic at $t=\infty$. Then, $n_{0} \neq n_{3}$.

Proof. We suppose that $n_{0}=n_{3}$. Especially, we treat the case where $n_{0}=n_{3}=1$ and show contradiction. If $n_{0}=n_{3}>1$, we can prove contradiction in the same way.

Comparing the coefficients of the terms $t^{2}, t$ in

$$
\begin{aligned}
& t q_{1}^{\prime}=2 q_{1}^{2} p_{1}-q_{1}^{2}+\left(\alpha_{0}+\alpha_{1}+\alpha_{3}\right) q_{1}-t+4 t p_{2}+2 q_{1} q_{2} p_{2}, \\
& t p_{1}^{\prime}=-2 q_{1} p_{1}^{2}+2 q_{1} p_{1}-\left(\alpha_{0}+\alpha_{1}+\alpha_{3}\right) p_{1}+\alpha_{0}-2 p_{1} q_{2} p_{2},
\end{aligned}
$$

we have

$$
2 a_{\infty, 1}^{2} b_{\infty, 0}-a_{\infty, 1}^{2}+4 d_{\infty, 1}+2 a_{\infty, 1} c_{\infty, 0} d_{\infty, 1}=0
$$




$$
-2 a_{\infty, 1} b_{\infty, 0}^{2}+2 a_{\infty, 1} b_{\infty, 0}-2 b_{\infty, 0} c_{\infty, 0} d_{\infty, 1}=0
$$

respectively.

Comparing the coefficients of the terms $t, t^{2}$ in

$$
\begin{aligned}
& t q_{2}^{\prime}=2 q_{2}^{2} p_{2}-q_{2}^{2}+\left(\alpha_{0}+\alpha_{1}+\alpha_{3}\right) q_{2}-t+4 t p_{1}+2 q_{1} p_{1} q_{2}, \\
& t p_{2}^{\prime}=-2 q_{2} p_{2}^{2}+2 q_{2} p_{2}-\left(\alpha_{0}+\alpha_{1}+\alpha_{3}\right) p_{2}+\alpha_{1}-2 q_{1} p_{1} p_{2},
\end{aligned}
$$

we obtain

$$
\begin{aligned}
& 2 c_{\infty, 0}^{2} d_{\infty, 1}-1+4 b_{\infty, 0}+2 a_{\infty, 1} b_{\infty, 0} c_{\infty, 0}=0 \\
& -2 c_{\infty, 0} d_{\infty, 1}^{2}-2 a_{\infty, 1} b_{\infty, 0} d_{\infty, 1}=0
\end{aligned}
$$

which implies that $b_{\infty, 0}=1 / 4$. Furthermore, from the second equation in (2.1) and the first equation in (2.2), it follows that $a_{\infty, 1}=0$, which is impossible.

Lemma 2.4. Suppose that for $A_{5}^{(2)}\left(\alpha_{j}\right)_{0 \leq j \leq 3}$, there exists a solution such that $q_{1}, p_{2}$ have both a pole at $t=\infty$ and $p_{1}, q_{2}$ are both holomorphic at $t=\infty$. Then, $n_{0}<n_{3}$.

Proposition 2.7. For $A_{5}^{(2)}\left(\alpha_{j}\right)_{0 \leq j \leq 3}$, there exists no solution such that $q_{1}, p_{2}$ have both a pole at $t=\infty$ and $p_{1}, q_{2}$ are both holomorphic at $t=\infty$.

Proof. We treat the case where $\left(n_{0}, n_{3}\right)=(1,2)$ and show contradiction. The other cases can be proved in the same way.

Comparing the coefficients of the terms $t^{3}$ in

$$
t q_{1}^{\prime}=2 q_{1}^{2} p_{1}-q_{1}^{2}+\left(\alpha_{0}+\alpha_{1}+\alpha_{3}\right) q_{1}-t+4 t p_{2}+2 q_{1} q_{2} p_{2}
$$

we have $d_{\infty, 2}=0$, which is impossible.

\subsubsection{The case where $p_{1}, p_{2}$ have a pole at $t=\infty$}

Proposition 2.8. For $A_{5}^{(2)}\left(\alpha_{j}\right)_{0 \leq j \leq 3}$, there exists no solution such that $p_{1}, p_{2}$ have both a pole at $t=\infty$ and $q_{1}, q_{2}$ are both holomorphic at $t=\infty$.

\subsection{The case where three of $\left(q_{1}, p_{1}, q_{2}, p_{2}\right)$ have a pole at $t=\infty$}

In this subsection, considering $\pi$, we treat the following two cases:

(1) $q_{1}, p_{1}, q_{2}$ all have a pole at $t=\infty$ and $p_{2}$ is holomorphic at $t=\infty$,

(2) $q_{1}, p_{1}, p_{2}$ all have a pole at $t=\infty$ and $q_{2}$ is holomorphic at $t=\infty$.

\subsubsection{The case where $q_{1}, p_{1}, q_{2}$ have a pole at $t=\infty$}

Proposition 2.9. For $A_{5}^{(2)}\left(\alpha_{j}\right)_{0 \leq j \leq 3}$, there exists no solution such that $q_{1}, p_{1}, q_{2}$ all have a pole at $t=\infty$ and $p_{2}$ is holomorphic at $t=\infty$. 


\subsubsection{The case where $q_{1}, p_{1}, p_{2}$ have a pole at $t=\infty$}

By Lemma 2.2, let us note that $q_{2} \not \equiv 0$.

Lemma 2.5. Suppose that for $A_{5}^{(2)}\left(\alpha_{j}\right)_{0 \leq j \leq 3}$, there exists a solution such that $q_{1}, p_{1}, p_{2}$ all have a pole at $t=\infty$ and $q_{2}$ is holomorphic at $t=\infty$. Moreover, assume that $q_{1}, p_{1}, p_{2}$ has a pole of order $n_{0}, n_{1}, n_{3}\left(n_{0}, n_{1}, n_{3} \geq 1\right)$ at $t=\infty$, respectively. Then, $n_{3} \geq n_{0}+n_{1}$.

Proof. Considering that

$$
t p_{1}^{\prime}=-2 q_{1} p_{1}^{2}+2 q_{1} p_{1}-\left(\alpha_{0}+\alpha_{1}+\alpha_{3}\right) p_{1}+\alpha_{0}-2 p_{1} q_{2} p_{2},
$$

we can prove the lemma.

Therefore, we define the nonnegative integer $k$ by $n_{3}=n_{0}+n_{1}+k$.

Lemma 2.6. Suppose that for $A_{5}^{(2)}\left(\alpha_{j}\right)_{0 \leq j \leq 3}$, there exists a solution such that $q_{1}, p_{1}, p_{2}$ all have a pole at $t=\infty$ and $q_{2}$ is holomorphic at $t=\infty$. Then, $c_{\infty, 0}=c_{\infty,-1}=\cdots=c_{\infty,-(k-1)}=0$, $a_{\infty, n_{0}} b_{\infty, 1}+c_{\infty,-k} d_{\infty, n_{3}}=0$, and $n_{0}-k \geq 1$

Proof. Considering that

$$
t p_{1}^{\prime}=-2 q_{1} p_{1}^{2}+2 q_{1} p_{1}-\left(\alpha_{0}+\alpha_{1}+\alpha_{3}\right) p_{1}+\alpha_{0}-2 p_{1} q_{2} p_{2},
$$

we find that $c_{\infty, 0}=c_{\infty,-1}=\cdots=c_{\infty,-(k-1)}=0, a_{\infty, n_{0}} b_{\infty, 1}+c_{\infty,-k} d_{\infty, n_{3}}=0$. Furthermore, considering that

$$
t q_{1}^{\prime}=2 q_{1}^{2} p_{1}-q_{1}^{2}+\left(\alpha_{0}+\alpha_{1}+\alpha_{3}\right) q_{1}-t+4 t p_{2}+2 q_{1} q_{2} p_{2},
$$

we can show the lemma.

Proposition 2.10. For $A_{5}^{(2)}\left(\alpha_{j}\right)_{0 \leq j \leq 3}$, there exists no solution such that $q_{1}, p_{1}, p_{2}$ all have a pole at $t=\infty$ and $q_{2}$ is holomorphic at $t=\infty$.

Proof. We treat the case where $n_{1}=1$. The other cases can be proved in the same way. Comparing the coefficients of the terms $t^{n_{0}+1}$ in

$$
t p_{1}^{\prime}=-2 q_{1} p_{1}^{2}+2 q_{1} p_{1}-\left(\alpha_{0}+\alpha_{1}+\alpha_{3}\right) p_{1}+\alpha_{0}-2 p_{1} q_{2} p_{2},
$$

we have

$$
-2 a_{\infty, n_{0}} b_{\infty, 0}-2 a_{\infty, n_{0}-1} b_{\infty, 1}+2 a_{\infty, n_{0}}-2 c_{\infty,-k} d_{\infty, n_{3}-1}-2 c_{\infty,-k-1} d_{\infty, n_{3}}=0 .
$$

If $n_{0}-k \geq 3$, comparing the coefficients of the terms $t^{2 n_{0}}$ in

$$
t q_{1}^{\prime}=2 q_{1}^{2} p_{1}-q_{1}^{2}+\left(\alpha_{0}+\alpha_{1}+\alpha_{3}\right) q_{1}-t+4 t p_{2}+2 q_{1} q_{2} p_{2},
$$

we obtain

$$
2 b_{\infty, 1} a_{\infty, n_{0}-1}+2 a_{\infty, n_{0}} b_{\infty, 0}-a_{\infty, n_{0}}+2 c_{\infty,-k} d_{\infty, n_{3}-1}+2 c_{\infty,-k-1} d_{\infty, n_{3}}=0 .
$$

Then, it follows that $a_{\infty, n_{0}}=0$, which is impossible.

If $n_{0}-k=2$, comparing the coefficients of the terms $t^{2}, t^{3 n_{0}-1}$ in

$$
\begin{aligned}
& t q_{2}^{\prime}=2 q_{2}^{2} p_{2}-q_{2}^{2}+\left(\alpha_{0}+\alpha_{1}+\alpha_{3}\right) q_{2}-t+4 t p_{1}+2 q_{1} p_{1} q_{2}, \\
& t p_{2}^{\prime}=-2 q_{2} p_{2}^{2}+2 q_{2} p_{2}-\left(\alpha_{0}+\alpha_{1}+\alpha_{3}\right) p_{2}+\alpha_{1}-2 q_{1} p_{1} p_{2},
\end{aligned}
$$


we have

$$
\begin{aligned}
& 2 d_{\infty, n_{3}} c_{\infty,-k} c_{\infty,-k-1}+2 d_{\infty, n_{3}-1} c_{\infty,-k}^{2}+4 b_{\infty, 1} \\
& \quad+2 c_{\infty,-k} a_{\infty, n_{0}} b_{\infty, 0}+2 c_{\infty,-k} a_{\infty, n_{0}-1} b_{\infty, 1}=0 \\
& -2 c_{\infty,-k} d_{\infty, n_{3}-1}-2 c_{\infty,-k-1} d_{\infty, n_{3}}-2 a_{\infty, n_{0}} b_{\infty, 0}-2 a_{\infty, n_{0}-1} b_{\infty, 1}=0
\end{aligned}
$$

respectively. Then, it follows that $b_{\infty, 1}=0$, which is impossible.

If $n_{0}-k=1$, comparing the coefficients of the terms $t^{2}$ in

$$
t q_{2}^{\prime}=2 q_{2}^{2} p_{2}-q_{2}^{2}+\left(\alpha_{0}+\alpha_{1}+\alpha_{3}\right) q_{2}-t+4 t p_{1}+2 q_{1} p_{1} q_{2},
$$

we obtain

$$
2 c_{\infty,-k}^{2} d_{\infty, n_{3}}+4 b_{\infty, 1}+2 a_{\infty, n_{0}} b_{\infty, 1} c_{\infty,-k}=4 b_{\infty, 1}=0
$$

which is impossible.

\subsection{The case where all of $\left(q_{1}, p_{1}, q_{2}, p_{2}\right)$ have a pole at $t=\infty$}

Lemma 2.7. Suppose that for $A_{5}^{(2)}\left(\alpha_{j}\right)_{0 \leq j \leq 3}$, there exists a solution such that $q_{1}, p_{1}, q_{2}, p_{2}$ all have a pole at $t=\infty$. Moreover, assume that $q_{1}, p_{1}, q_{2}, p_{2}$ have a pole of order $n_{0}, n_{1}, n_{2}, n_{3}$ $\left(n_{0}, n_{1}, n_{2}, n_{3} \geq 1\right)$ at $t=\infty$, respectively. Then, $n_{0}+n_{1}=n_{2}+n_{3}$.

Proof. Considering

$$
t p_{1}^{\prime}=-2 q_{1} p_{1}^{2}+2 q_{1} p_{1}-\left(\alpha_{0}+\alpha_{1}+\alpha_{3}\right) p_{1}+\alpha_{0}-2 p_{1} q_{2} p_{2},
$$

we can show the lemma.

Therefore, we see that $n_{0}+n_{1}=n_{2}+n_{3} \geq 2$.

Proposition 2.11. For $A_{5}^{(2)}\left(\alpha_{j}\right)_{0 \leq j \leq 3}$, there exists no solution such that $q_{1}, p_{1}, q_{2}, p_{2}$ all have a pole at $t=\infty$.

Proof. We treat the case where $n_{0}+n_{1}=n_{2}+n_{3}=2$. The other cases can be proved in the same way.

Comparing the coefficients of the term $t^{3}$ in

$$
t p_{1}^{\prime}=-2 q_{1} p_{1}^{2}+2 q_{1} p_{1}-\left(\alpha_{0}+\alpha_{1}+\alpha_{3}\right) p_{1}+\alpha_{0}-2 p_{1} q_{2} p_{2},
$$

we have $a_{\infty, 1} b_{\infty, 1}+c_{\infty, 1} d_{\infty, 1}=0$.

Comparing the coefficients of the term $t^{2}$ in

$$
\begin{aligned}
& t q_{1}^{\prime}=2 q_{1}^{2} p_{1}-q_{1}^{2}+\left(\alpha_{0}+\alpha_{1}+\alpha_{3}\right) q_{1}-t+4 t p_{2}+2 q_{1} q_{2} p_{2}, \\
& t p_{1}^{\prime}=-2 q_{1} p_{1}^{2}+2 q_{1} p_{1}-\left(\alpha_{0}+\alpha_{1}+\alpha_{3}\right) p_{1}+\alpha_{0}-2 p_{1} q_{2} p_{2}, \\
& t q_{2}^{\prime}=2 q_{2}^{2} p_{2}-q_{2}^{2}+\left(\alpha_{0}+\alpha_{1}+\alpha_{3}\right) q_{2}-t+4 t p_{1}+2 q_{1} p_{1} q_{2}, \\
& t p_{2}^{\prime}=-2 q_{2} p_{2}^{2}+2 q_{2} p_{2}-\left(\alpha_{0}+\alpha_{1}+\alpha_{3}\right) p_{2}+\alpha_{1}-2 q_{1} p_{1} p_{2},
\end{aligned}
$$

we obtain

$$
\begin{aligned}
& 2 a_{\infty, 1} a_{\infty, 0} b_{\infty, 1}+2 b_{\infty, 0} a_{\infty, 1}^{2}-a_{\infty, 1}^{2}+4 d_{\infty, 1}+2 a_{\infty, 1} c_{\infty, 1} d_{\infty, 0}+2 a_{\infty, 1} c_{\infty, 0} d_{\infty, 1}=0, \\
& -2 a_{\infty, 1} b_{\infty, 0}-2 a_{\infty, 0} b_{\infty, 1}+2 a_{\infty, 1}-2 c_{\infty, 1} d_{\infty, 0}-2 c_{\infty, 0} d_{\infty, 1}=0 \\
& 2 c_{\infty, 1} c_{\infty, 0} d_{\infty, 1}+2 d_{\infty, 0} c_{\infty, 1}^{2}-c_{\infty, 1}^{2}+4 b_{\infty, 1}+2 c_{\infty, 1} a_{\infty, 1} b_{\infty, 0}+2 c_{\infty, 1} a_{\infty, 0} b_{\infty, 1}=0, \\
& -2 c_{\infty, 1} d_{\infty, 0}-2 c_{\infty, 0} d_{\infty, 1}+2 c_{\infty, 1}-2 a_{\infty, 1} b_{\infty, 0}-2 a_{\infty, 0} b_{\infty, 1}=0
\end{aligned}
$$


respectively. Based on the second and fourth equations of (2.3), we have $a_{\infty, 1}=c_{\infty, 1}$. From the first and second equations of (2.3), we obtain $a_{\infty, 1}^{2}+4 d_{\infty, 1}=0$. From the third and fourth equations of (2.3), we have $c_{\infty, 1}^{2}+4 b_{\infty, 1}=0$.

Therefore, since $a_{\infty, 1} b_{\infty, 1}+c_{\infty, 1} d_{\infty, 1}=0$, it follows that $a_{\infty, 1} b_{\infty, 1}=0$, which is impossible.

\subsection{Summary}

Proposition 2.12. For $A_{5}^{(2)}\left(\alpha_{j}\right)_{0 \leq j \leq 3}$, there exists a meromorphic solution at $t=\infty$. Then, $q_{1}, p_{1}, q_{2}, p_{2}$ are uniquely expanded as follows:

$$
\left\{\begin{array}{l}
q_{1}=\left(-2 \alpha_{0}+\alpha_{3}\right)+\cdots, \\
p_{1}=1 / 4+\left(-2 \alpha_{1}+\alpha_{3}\right)\left(-2 \alpha_{1}-\alpha_{3}\right) t^{-1} / 4+\cdots, \\
q_{2}=\left(-2 \alpha_{1}+\alpha_{3}\right)+\cdots, \\
p_{2}=1 / 4+\left(-2 \alpha_{0}+\alpha_{3}\right)\left(-2 \alpha_{0}-\alpha_{3}\right) t^{-1} / 4+\cdots .
\end{array}\right.
$$

\section{Meromorphic solution at $t=0$}

In this section, we treat meromorphic solutions at $t=0$. Then, in the same way as Proposition 2.12, we can show the following proposition:

Proposition 3.1. Suppose that for $A_{5}^{(2)}\left(\alpha_{j}\right)_{0 \leq j \leq 3}$, there exists a meromorphic solution at $t=0$. Then, one of the following occurs:

(1) $q_{1}, p_{1}, q_{2}, p_{2}$ are all holomorphic at $t=0$,

(2) $p_{1}$ has a pole of order one at $t=0$ and $q_{1}, q_{2}, p_{2}$ are all holomorphic at $t=0$,

(3) $p_{2}$ has a pole of order one at $t=0$ and $q_{1}, p_{1}, q_{2}$ are all holomorphic at $t=0$.

In this paper, we define the coefficients of the Lauren series of $q_{1}, p_{1}, q_{2}, p_{2}$ at $t=0$ by $a_{0, k}$, $b_{0, k}, c_{0, k}, d_{0, k}, k \in \mathbb{Z}$. In this section, we prove that the constant terms of $q_{1}, q_{2}$ at $t=0, a_{0,0}$, $c_{0,0}$ are zero, or expressed by the parameters, $\alpha_{j}(0 \leq j \leq 3)$.

\subsection{The case where $q_{1}, p_{1}, q_{2}, p_{2}$ are all holomorphic at $t=0$}

Proposition 3.2. Suppose that for $A_{5}^{(2)}\left(\alpha_{j}\right)_{0 \leq j \leq 3}$, there exists a solution such that $q_{1}, p_{1}, q_{2}$, $p_{2}$ are all holomorphic at $t=0$. Then, one of the following occurs:

(1) $a_{0,0}=0,-\left(\alpha_{0}+\alpha_{1}+\alpha_{3}\right) b_{0,0}+\alpha_{0}=0, c_{0,0}=0,-\left(\alpha_{0}+\alpha_{1}+\alpha_{3}\right) d_{0,0}+\alpha_{1}=0$,

(2) $a_{0,0}=0,\left(-\alpha_{0}+\alpha_{1}-\alpha_{3}\right) b_{0,0}+\alpha_{0}=0, c_{0,0}=\alpha_{0}-\alpha_{1}+\alpha_{3},\left(-\alpha_{0}+\alpha_{1}-\alpha_{3}\right) d_{0,0}-\alpha_{1}=0$,

(3) $a_{0,0}=-\alpha_{0}+\alpha_{1}+\alpha_{3},\left(\alpha_{0}-\alpha_{1}-\alpha_{3}\right) b_{0,0}-\alpha_{0}=0, c_{0,0}=0,\left(\alpha_{0}-\alpha_{1}-\alpha_{3}\right) d_{0,0}+\alpha_{1}=0$,

(4) $a_{0,0}=-\alpha_{0}-\alpha_{1}+\alpha_{3},\left(\alpha_{0}+\alpha_{1}-\alpha_{3}\right) b_{0,0}-\alpha_{0}=0, c_{0,0}=-\alpha_{0}-\alpha_{1}+\alpha_{3}$, $\left(\alpha_{0}+\alpha_{1}-\right.$ $\left.\alpha_{3}\right) d_{0,0}-\alpha_{1}=0$.

\subsection{The case where $p_{1}$ has a pole at $t=0$}

Proposition 3.3. Suppose that for $A_{5}^{(2)}\left(\alpha_{j}\right)_{0 \leq j \leq 3}$, there exists a solution such that $p_{1}$ has a pole at $t=0$ and $q_{1}, q_{2}, p_{2}$ are all holomorphic at $t=0$. Then,

$$
\left\{\begin{array}{l}
q_{1}=\left(-8 \alpha_{0}-8 \alpha_{3}+6\right) t /\left\{\left(4 \alpha_{1}-1\right)\left(4 \alpha_{1}+1\right)\right\}+\cdots, \\
p_{1}=\left(4 \alpha_{1}-1\right)\left(4 \alpha_{1}+1\right) t^{-1} / 16+\cdots, \\
q_{2}=\left(-2 \alpha_{1}+1 / 2\right)+\cdots \\
p_{2}=1 / 4+\cdots
\end{array}\right.
$$




\subsection{The case where $p_{2}$ has a pole at $t=0$}

Proposition 3.4. Suppose that for $A_{5}^{(2)}\left(\alpha_{j}\right)_{0 \leq j \leq 3}$, there exists a solution such that $p_{2}$ has a pole at $t=0$ and $q_{1}, p_{1}, q_{2}$ are all holomorphic at $t=0$. Then,

$$
\left\{\begin{array}{l}
q_{1}=\left(-2 \alpha_{0}+1 / 2\right)+\cdots \\
p_{1}=1 / 4+\cdots \\
q_{2}=\left(-8 \alpha_{1}-8 \alpha_{3}+6\right) t /\left\{\left(4 \alpha_{0}-1\right)\left(4 \alpha_{0}+1\right)\right\}+\cdots \\
p_{2}=\left(4 \alpha_{0}-1\right)\left(4 \alpha_{0}+1\right) t^{-1} / 16+\cdots
\end{array}\right.
$$

\section{Meromorphic solution at $t=c \in \mathbb{C}^{*}$}

In this section, we deal with meromorphic solutions at $t=c \in \mathbb{C}^{*}$, where $\mathbb{C}^{*}$ means the set of nonzero complex numbers.

Proposition 4.1. Suppose that for $A_{5}^{(2)}\left(\alpha_{j}\right)_{0 \leq j \leq 3}$, there exists a meromorphic solution at $t=$ $c \in \mathbb{C}^{*}$ such that some of $\left(q_{1}, p_{1}, q_{2}, p_{2}\right)$ have a pole at $t=c$. Then, one of the following occurs:

(1) $q_{1}$ has a pole at $t=c$ and $p_{1}, q_{2}, p_{2}$ are all holomorphic at $t=c$,

(2) $q_{2}$ has a pole at $t=c$ and $q_{1}, p_{1}, p_{2}$ are all holomorphic at $t=c$,

(3) $q_{1}, q_{2}$ have both a pole at $t=c$ and $p_{1}, p_{2}$ are both holomorphic at $t=c$,

(4) $q_{1}, p_{2}$ have both a pole at $t=c$ and $p_{1}, q_{2}$ are both holomorphic at $t=c$,

(5) $p_{1}, q_{2}$ have both a pole at $t=c$ and $q_{1}, p_{2}$ are both holomorphic at $t=c$,

(6) $p_{1}, p_{2}$ have both a pole at $t=c$ and $q_{1}, q_{2}$ are both holomorphic at $t=c$,

(7) $q_{1}, p_{1}, q_{2}, p_{2}$ all have a pole at $t=c$.

\subsection{The case where $q_{1}$ has a pole at $t=c \in \mathbb{C}^{*}$}

Proposition 4.2. Suppose that for $A_{5}^{(2)}\left(\alpha_{j}\right)_{0 \leq j \leq 3}$, there exists a solution such that $q_{1}$ has a pole at $t=c \in \mathbb{C}^{*}$ and $p_{1}, q_{2}, p_{2}$ are all holomorphic at $t=c$. Then, either of the following occurs:
(1) $\left\{\begin{array}{l}q_{1}=c(t-c)^{-1}+\cdots \\ p_{1}=-\frac{\alpha_{0}}{c}(t-c)+\cdots\end{array}\right.$
$\left\{\begin{array}{l}q_{1}=-c(t-c)^{-1}+\cdots \\ p_{1}=1+\frac{\alpha_{1}+\alpha_{3}}{c}(t-c)+\cdots \\ q_{2}=O(t-c) \\ p_{2}=O(t-c)\end{array}\right.$

\subsection{The case where $q_{2}$ has a pole at $t=c \in \mathbb{C}^{*}$}

Proposition 4.3. Suppose that for $A_{5}^{(2)}\left(\alpha_{j}\right)_{0 \leq j \leq 3}$, there exists a solution such that $q_{2}$ has a pole at $t=c \in \mathbb{C}^{*}$ and $q_{1}, p_{1}, p_{2}$ are all holomorphic at $t=c$. Then, either of the following occurs:
(1) $\left\{\begin{array}{l}q_{2}=c(t-c)^{-1}+\cdots \\ p_{2}=-\frac{\alpha_{1}}{c}(t-c)+\cdots\end{array}\right.$
$(2)\left\{\begin{array}{l}q_{1}=O(t-c) \\ p_{1}=O(t-c) \\ q_{2}=-c(t-c)^{-1}+\cdots \\ p_{2}=1+\frac{\alpha_{0}+\alpha_{3}}{c}(t-c)+\cdots\end{array}\right.$ 


\subsection{The case where $q_{1}, q_{2}$ have a pole at $t=c \in \mathbb{C}^{*}$}

Proposition 4.4. Suppose that for $A_{5}^{(2)}\left(\alpha_{j}\right)_{0 \leq j \leq 3}$, there exists a solution such that $q_{1}$, $q_{2}$ have both a pole at $t=c \in \mathbb{C}^{*}$ and $p_{1}, p_{2}$ are both holomorphic at $t=c$. Then, either of the following occurs:

(1)

$$
\left\{\begin{array}{l}
q_{1}=-c(t-c)^{-1}+\cdots \\
p_{1}=b_{c, 0}+b_{c, 1}(t-c)+\cdots \\
q_{2}=-c(t-c)^{-1}+\cdots \\
p_{2}=d_{c, 0}+d_{c, 1}(t-c)+\cdots
\end{array}\right.
$$

$$
\left\{\begin{array}{l}
q_{1}=c(t-c)^{-1}+\cdots \\
p_{1}=-\frac{\alpha_{0}}{c}(t-c)+\cdots \\
q_{2}=c(t-c)^{-1}+\cdots \\
p_{2}=-\frac{\alpha_{1}}{c}(t-c)+\cdots
\end{array}\right.
$$

where $b_{c, 0}+d_{c, 0}=1$ and $b_{c, 1}+d_{c, 1}=\frac{\alpha_{3}}{c}$.

\subsection{The case where $q_{1}, p_{2}$ have a pole at $t=c \in \mathbb{C}^{*}$}

Proposition 4.5. Suppose that for $A_{5}^{(2)}\left(\alpha_{j}\right)_{0 \leq j \leq 3}$, there exists a solution such that $q_{1}$, $p_{2}$ have both a pole at $t=c \in \mathbb{C}^{*}$ and $p_{1}, q_{2}$ are both holomorphic at $t=c$. Then, one of the following occurs:

$$
\begin{gathered}
(1)\left\{\begin{array}{l}
q_{1}=4 c(t-c)^{-1}+8 / 3+\cdots, \\
p_{1}=0-\alpha_{0} /\{5 c\} \cdot(t-c)+\cdots, \\
q_{2}=(t-c)+\left(3 \alpha_{0}-\alpha_{1}-\alpha_{3}+2\right) /\{2 c\} \cdot(t-c)^{2}+\cdots, \\
p_{2}=c(t-c)^{-2}-4 \alpha_{0} / 5 \cdot(t-c)^{-1}+\cdots,
\end{array}\right. \\
(2)\left\{\begin{array}{l}
q_{1}=-c(t-c)^{-1}+\left(-1 / 4-\alpha_{0}\right)+\cdots, \\
p_{1}=0+\alpha_{0} /\{5 c\} \cdot(t-c) \\
q_{2}=(t-c)+\left(-3 \alpha_{0}-\alpha_{1}-\alpha_{3}+2\right) /\{2 c\} \cdot(t-c)^{2}+\cdots, \\
p_{2}=c(t-c)^{-2}+4 \alpha_{0} / 5 \cdot(t-c)^{-1}+\cdots,
\end{array}\right. \\
\text { (3) }\left\{\begin{array}{l}
q_{1}=c(t-c)^{-1}+\left(3 / 4-\alpha_{0}\right)+\cdots, \\
p_{1}=1 / 2-1 /\{12 c\} \cdot(t-c)+\cdots, \\
q_{2}=-(t-c)+\left[\left(\alpha_{1}+\alpha_{3}\right) / c-3 /\{4 c\}\right](t-c)^{2}+\cdots, \\
p_{2}=-c / 2 \cdot(t-c)^{-2}-1 / 6(t-c)^{-1}+\cdots
\end{array}\right.
\end{gathered}
$$

\subsection{The case where $p_{1}, q_{2}$ have a pole at $t=c \in \mathbb{C}^{*}$}

Proposition 4.6. Suppose that for $A_{5}^{(2)}\left(\alpha_{j}\right)_{0 \leq j \leq 3}$, there exists a solution such that $p_{1}, q_{2}$ have both a pole at $t=c \in \mathbb{C}^{*}$ and $q_{1}, p_{2}$ are both holomorphic at $t=c$. Then, one of the following occurs:

$$
\begin{aligned}
& \left\{\begin{array}{l}
q_{1}=(t-c)+\left(3 \alpha_{1}-\alpha_{0}-\alpha_{3}+2\right) /\{2 c\} \cdot(t-c)^{2}+\cdots \\
p_{1}=c(t-c)^{-2}-4 \alpha_{1} / 5 \cdot(t-c)^{-1}+\cdots \\
q_{2}=4 c(t-c)^{-1}+8 / 3+\cdots \\
p_{2}=0-\alpha_{1} /\{5 c\} \cdot(t-c)+\cdots
\end{array}\right. \\
& \left\{\begin{array}{l}
q_{1}=(t-c)+\left(-3 \alpha_{1}-\alpha_{0}-\alpha_{3}+2\right) /\{2 c\} \cdot(t-c)^{2}+\cdots \\
p_{1}=c(t-c)^{-2}+4 \alpha_{1} / 5 \cdot(t-c)^{-1}+\cdots \\
q_{2}=-c(t-c)^{-1}+\left(-1 / 4-\alpha_{1}\right)+\cdots \\
p_{2}=0+\alpha_{1} /\{5 c\} \cdot(t-c)+\cdots
\end{array}\right.
\end{aligned}
$$


(3) $\left\{\begin{array}{l}q_{1}=-(t-c)+\left[\left(\alpha_{0}+\alpha_{3}\right) / c-3 /\{4 c\}\right](t-c)^{2}+\cdots \\ p_{1}=-c / 2 \cdot(t-c)^{-2}-1 / 6(t-c)^{-1}+\cdots \\ q_{2}=c(t-c)^{-1}+\left(3 / 4-\alpha_{1}\right)+\cdots \\ p_{2}=1 / 2-1 /\{12 c\} \cdot(t-c)+\cdots\end{array}\right.$

\subsection{The case where $p_{1}, p_{2}$ have a pole at $t=c \in \mathbb{C}^{*}$}

Proposition 4.7. Suppose that for $A_{5}^{(2)}\left(\alpha_{j}\right)_{0 \leq j \leq 3}$, there exists a solution such that $p_{1}, p_{2}$ have both a pole at $t=c \in \mathbb{C}^{*}$ and $q_{1}, q_{2}$ are both holomorphic at $t=c$. Then,

$$
\left\{\begin{array}{l}
q_{1}=\left(-4 d_{c,-1}\right)+a_{c, 1}(t-c)+\cdots \\
p_{1}=b_{c,-1}(t-c)^{-1}+\left(3 / 8+2 b_{c,-1}^{2} / c\right)+\cdots, \\
q_{2}=\left(-4 b_{c,-1}\right)+c_{c, 1}(t-c)+\cdots \\
p_{2}=d_{c,-1}(t-c)^{-1}+\left(3 / 8+2 d_{c,-1}^{2} / c\right)+\cdots,
\end{array}\right.
$$

where the coefficients satisfy

$$
16 b_{c,-1} d_{c,-1}+c=0, \quad a_{c, 1} b_{c,-1} c+c_{c, 1} d_{c,-1} c+\frac{c}{2}\left(\alpha_{0}+\alpha_{1}+\alpha_{3}\right)=\frac{c}{2} .
$$

\subsection{The case where $q_{1}, p_{1}, q_{2}, p_{2}$ have a pole at $t=c \in \mathbb{C}^{*}$}

Proposition 4.8. Suppose that for $A_{5}^{(2)}\left(\alpha_{j}\right)_{0 \leq j \leq 3}$, there exists a solution such that $q_{1}, p_{1}, q_{2}$, $p_{2}$ all have a pole at $t=c \in \mathbb{C}^{*}$. Then,

$$
\left\{\begin{array}{l}
q_{1}=-2 c(t-c)^{-1}+(\sqrt{c}-4 / 3)+a_{c, 1}(t-c)+\cdots, \\
p_{1}=\sqrt{c} / 4 \cdot(t-c)^{-1}+1 / 2+b_{c, 1}(t-c)+\cdots, \\
q_{2}=-2 c(t-c)^{-1}+(-\sqrt{c}-4 / 3)+c_{c, 1}(t-c)+\cdots, \\
p_{2}=-\sqrt{c} / 4 \cdot(t-c)^{-1}+1 / 2+d_{c, 1}(t-c)+\cdots,
\end{array}\right.
$$

where the coefficients satisfy

$$
b_{c, 1}+d_{c, 1}=\alpha_{3} /\{2 c\}, \quad a_{c, 1} \sqrt{c}-c_{c, 1} \sqrt{c}=2+2 \alpha_{3}-2 \alpha_{0}-2 \alpha_{1} .
$$

\subsection{Summary}

\section{Proposition 4.9.}

(1) Suppose that for $A_{5}^{(2)}\left(\alpha_{j}\right)_{0 \leq j \leq 3}$, there exists a meromorphic solution at $t=c \in \mathbb{C}^{*}$. Then, $q_{1}, q_{2}$ have both a pole of order at most one at $t=c$ and the residues of $q_{1}, q_{2}$ at $t=c$ are expressed by $n c(n \in \mathbb{Z})$.

(2) Suppose that for $A_{5}^{(2)}\left(\alpha_{j}\right)_{0 \leq j \leq 3}$, there exists a rational solution. Then, $a_{\infty, 0}-a_{0,0} \in \mathbb{Z}$, $c_{\infty, 0}-c_{0,0} \in \mathbb{Z}$.

Proof. Case (1) is obvious. Let us prove case (2). From the discussions in Sections 2, 3 and 4, it follows that

$$
q_{1}=a_{\infty, 0}+\sum_{j=1}^{m_{1}} \frac{n_{j} c_{j}}{t-c_{j}}, \quad q_{2}=c_{\infty, 0}+\sum_{k=1}^{m_{2}} \frac{n_{k}^{\prime} c_{k}^{\prime}}{t-c_{k}^{\prime}}, \quad n_{j}, n_{k}^{\prime} \in \mathbb{Z},
$$

where $m_{1}, m_{2}$ are both positive integers and $c_{k} \in \mathbb{C}^{*}\left(1 \leq k \leq m_{1}\right)$ and $c_{j}^{\prime} \in \mathbb{C}^{*}\left(1 \leq j \leq m_{2}\right)$ are poles of $q_{1}$ and $q_{2}$, respectively. If $q_{1}$ or $q_{2}$ is holomorphic in $\mathbb{C}^{*}$, then its second sum is considered to be zero.

Considering the constant terms of the Taylor series of $q_{1}, q_{2}$ at $t=0$, we can prove the proposition. 


\section{The Laurent series of the Hamiltonian $H$}

In this section, for a meromorphic solution at $t=\infty, 0$, we first compute the constant terms $h_{\infty, 0}, h_{0,0}$ of the Laurent series of the Hamiltonian $H$ at $t=\infty, 0$. Moreover, for a meromorphic solution at $t=c \in \mathbb{C}^{*}$, we calculate the residue of $H$ at $t=c$.

\subsection{The Laurent series of $H$ at $t=\infty$}

Proposition 5.1. Suppose that for $A_{5}^{(2)}\left(\alpha_{j}\right)_{0 \leq j \leq 3}$, there exists a meromorphic solution at $t=\infty$. Then,

$$
h_{\infty, 0}=\frac{3}{4}\left(\alpha_{0}+\alpha_{1}+\alpha_{3}\right)^{2}-\frac{1}{2}\left(-2 \alpha_{0}+\alpha_{3}\right)\left(-2 \alpha_{1}+\alpha_{3}\right)-3\left(\alpha_{0}+\alpha_{1}\right) \alpha_{3} .
$$

\subsection{The Laurent series of $H$ at $t=0$}

\subsubsection{The case where $q_{1}, p_{1}, q_{2}, p_{2}$ are all holomorphic at $t=0$}

Proposition 5.2. Suppose that for $A_{5}^{(2)}\left(\alpha_{j}\right)_{0 \leq j \leq 3}$, there exists a solution such that $q_{1}, p_{1}, q_{2}$, $p_{2}$ are all holomorphic at $t=0$. Then,

$$
h_{0,0}= \begin{cases}0 & \text { if case (1) occurs in Proposition 3.2, } \\ -\alpha_{1}\left(\alpha_{0}+\alpha_{3}\right) & \text { if case (2) occurs in Proposition 3.2, } \\ -\alpha_{0}\left(\alpha_{1}+\alpha_{3}\right) & \text { if case (3) occurs in Proposition 3.2, } \\ -\alpha_{3}\left(\alpha_{0}+\alpha_{1}\right) & \text { if case (4) occurs in Proposition 3.2. }\end{cases}
$$

5.2.2 The case where $p_{1}$ has a pole at $t=0$

Proposition 5.3. Suppose that for $A_{5}^{(2)}\left(\alpha_{j}\right)_{0 \leq j \leq 3}$, there exists a solution such that $p_{1}$ has a pole at $t=0$ and $q_{1}, q_{2}, p_{2}$ are all holomorphic at $t=0$. Then,

$$
h_{0,0}=-\frac{1}{4}\left(\alpha_{0}+\alpha_{1}+\alpha_{3}\right)^{2}+\alpha_{1}^{2}+\frac{3}{16} \text {. }
$$

\subsubsection{The case where $p_{2}$ has a pole at $t=0$}

Proposition 5.4. Suppose that for $A_{5}^{(2)}\left(\alpha_{j}\right)_{0 \leq j \leq 3}$, there exists a solution such that $p_{2}$ has a pole at $t=0$ and $q_{1}, p_{1}, q_{2}$ are all holomorphic at $t=0$. Then,

$$
h_{0,0}=-\frac{1}{4}\left(\alpha_{0}+\alpha_{1}+\alpha_{3}\right)^{2}+\alpha_{0}^{2}+\frac{3}{16} \text {. }
$$

\subsection{The Laurent series of $H$ at $t=c \in \mathbb{C}^{*}$}

5.3.1 The case where $q_{1}$ has a pole at $t=c \in \mathbb{C}^{*}$

Proposition 5.5. Suppose that for $A_{5}^{(2)}\left(\alpha_{j}\right)_{0 \leq j \leq 3}$, there exists a solution such that $q_{1}$ has a pole at $t=c \in \mathbb{C}^{*}$ and $p_{1}, q_{2}, p_{2}$ are all holomorphic at $t=c$. Then, $H$ is holomorphic at $t=c$.

\subsubsection{The case where $q_{2}$ has a pole at $t=c \in \mathbb{C}^{*}$}

Proposition 5.6. Suppose that for $A_{5}^{(2)}\left(\alpha_{j}\right)_{0 \leq j \leq 3}$, there exists a solution such that $q_{2}$ has a pole at $t=c \in \mathbb{C}^{*}$ and $q_{1}, p_{1}, p_{2}$ are all holomorphic at $t=c$. Then, $H$ is holomorphic at $t=c$. 


\subsubsection{The case where $q_{1}, q_{2}$ have a pole at $t=c \in \mathbb{C}^{*}$}

Proposition 5.7. Suppose that for $A_{5}^{(2)}\left(\alpha_{j}\right)_{0 \leq j \leq 3}$, there exists a solution such that $q_{1}$, $q_{2}$ have both a pole at $t=c \in \mathbb{C}^{*}$ and $p_{1}, p_{2}$ are both holomorphic at $t=c$. Then, $H$ is holomorphic at $t=c$.

\subsubsection{The case where $q_{1}, p_{2}$ have a pole at $t=c \in \mathbb{C}^{*}$}

Proposition 5.8. Suppose that for $A_{5}^{(2)}\left(\alpha_{j}\right)_{0 \leq j \leq 3}$, there exists a solution such that $q_{1}$, $q_{2}$ have both a pole at $t=c \in \mathbb{C}^{*}$ and $p_{1}, p_{2}$ are both holomorphic at $t=c$. Then, $H$ has a pole of order one at $t=c$ and

$$
\underset{t=c}{\operatorname{Res}} H= \begin{cases}c & \text { if case }(1) \text { occurs in Proposition 4.5, } \\ c & \text { if case (2) occurs in Proposition 4.5, } \\ c / 2 & \text { if case (3) occurs in Proposition 4.5. }\end{cases}
$$

\subsubsection{The case where $p_{1}, q_{2}$ have a pole at $t=c \in \mathbb{C}^{*}$}

Proposition 5.9. Suppose that for $A_{5}^{(2)}\left(\alpha_{j}\right)_{0 \leq j \leq 3}$, there exists a solution such that $p_{1}$, $q_{2}$ have both a pole at $t=c \in \mathbb{C}^{*}$ and $q_{1}, p_{2}$ are both holomorphic at $t=c$. Then, $H$ has a pole of order one at $t=c$ and

$$
\operatorname{Res}_{t=c} H= \begin{cases}c & \text { if case }(1) \text { occurs in Proposition 4.6, } \\ c & \text { if case }(2) \text { occurs in Proposition 4.6, } \\ c / 2 & \text { if case }(3) \text { occurs in Proposition 4.6. }\end{cases}
$$

\subsubsection{The case where $p_{1}, p_{2}$ have a pole at $t=c \in \mathbb{C}^{*}$}

Proposition 5.10. Suppose that for $A_{5}^{(2)}\left(\alpha_{j}\right)_{0 \leq j \leq 3}$, there exists a solution such that $p_{1}, p_{2}$ have both a pole at $t=c \in \mathbb{C}^{*}$ and $q_{1}, q_{2}$ are both holomorphic at $t=c$. Then, $H$ has a pole of order one at $t=c$ and $\operatorname{Res}_{t=c} H=c / 4$.

\subsubsection{The case where $q_{1}, p_{1}, q_{2}, p_{2}$ have a pole at $t=c \in \mathbb{C}^{*}$}

Proposition 5.11. Suppose that for $A_{5}^{(2)}\left(\alpha_{j}\right)_{0 \leq j \leq 3}$, there exists a solution such that $q_{1}, p_{1}, q_{2}$, $p_{2}$ all have a pole at $t=c \in \mathbb{C}^{*}$. Then, $H$ has a pole of order one at $t=c$ and $\operatorname{Res}_{t=c} H=c / 4$.

\subsection{Summary}

\section{Proposition 5.12.}

(1) Suppose that for $A_{5}^{(2)}\left(\alpha_{j}\right)_{0 \leq j \leq 3}$, there exists a meromorphic solution at $t=c \in \mathbb{C}^{*}$. Then, the residue of $H$ at $t=c$ is expressed by $n c / 4(n \in \mathbb{Z})$.

(2) Suppose that for $A_{5}^{(2)}\left(\alpha_{j}\right)_{0 \leq j \leq 3}$, there exists a rational solution. Then, $4\left(h_{\infty, 0}-h_{0,0}\right) \in \mathbb{Z}$.

Proof. Case (1) is obvious. Case (2) can be proved in the same way as Proposition 4.9. 


\section{Necessary condition ... (1)}

\subsection{The case where $q_{1}, p_{1}, q_{2}, p_{2}$ are all holomorphic at $t=0$}

\subsubsection{The case where $a_{0,0}=0, c_{0,0}=0$}

Proposition 6.1. Suppose that for $A_{5}^{(2)}\left(\alpha_{j}\right)_{0 \leq j \leq 3}$, there exists a rational solution such that $q_{1}$, $p_{1}, q_{2}, p_{2}$ are all holomorphic at $t=0$. Moreover, assuming that $a_{0,0}=0, c_{0,0}=0$, then, $-2 \alpha_{0}+\alpha_{3} \in \mathbb{Z},-2 \alpha_{1}+\alpha_{3} \in \mathbb{Z}$.

Proof. The proposition follows from Propositions 2.12, 4.9.

\subsubsection{The case where $a_{0,0}=0, c_{0,0} \neq 0$}

Proposition 6.2. Suppose that for $A_{5}^{(2)}\left(\alpha_{j}\right)_{0 \leq j \leq 3}$, there exists a rational solution such that $q_{1}$, $p_{1}, q_{2}, p_{2}$ are all holomorphic at $t=0$. Moreover, assuming that $a_{0,0}=0, c_{0,0} \neq 0$, then, $-2 \alpha_{0}+\alpha_{3} \in \mathbb{Z}, 2 \alpha_{1}+\alpha_{3} \in \mathbb{Z}$.

Proof. The proposition follows from Propositions 2.12, 3.2 and 4.9.

\subsubsection{The case where $a_{0,0} \neq 0, c_{0,0}=0$}

Proposition 6.3. Suppose that for $A_{5}^{(2)}\left(\alpha_{j}\right)_{0 \leq j \leq 3}$, there exists a rational solution such that $q_{1}$, $p_{1}, q_{2}, p_{2}$ are all holomorphic at $t=0$. Moreover, assuming that $a_{0,0} \neq 0, c_{0,0}=0$, then, $2 \alpha_{0}+\alpha_{3} \in \mathbb{Z},-2 \alpha_{1}+\alpha_{3} \in \mathbb{Z}$.

Proof. The proposition follows from Propositions 2.12, 3.2 and 4.9.

\subsubsection{The case where $a_{0,0} \neq 0, c_{0,0} \neq 0$}

Proposition 6.4. Suppose that for $A_{5}^{(2)}\left(\alpha_{j}\right)_{0 \leq j \leq 3}$, there exists a rational solution such that $q_{1}$, $p_{1}, q_{2}, p_{2}$ are all holomorphic at $t=0$. Moreover, assuming that $a_{0,0} \neq 0, c_{0,0} \neq 0$, then, $2 \alpha_{0}+\alpha_{3} \in \mathbb{Z}, 2 \alpha_{1}+\alpha_{3} \in \mathbb{Z}$.

Proof. From Propositions 2.12, 3.2 and 4.9, it follows that $\alpha_{0}-\alpha_{1} \in \mathbb{Z}$.

If $\alpha_{0} \neq 0$, by Proposition 3.2, we find that $s_{0}\left(q_{1}, p_{1}, q_{2}, p_{2}\right)$ is a rational solution of $A_{5}^{(2)}\left(-\alpha_{0}\right.$, $\left.\alpha_{1}, \alpha_{2}+\alpha_{0}, \alpha_{3}\right)$ such that all of $s_{0}\left(q_{1}, p_{1}, q_{2}, p_{2}\right)$ are holomorphic at $t=0$ and $a_{0,0}=0, c_{0,0} \neq 0$. Then, from Proposition 6.2, we obtain the necessary condition. If $\alpha_{1} \neq 0$, by $s_{1}$ and Proposition 6.3 , we obtain the necessary condition in the same way.

If $\alpha_{0}=\alpha_{1}=0$ and $\alpha_{2} \neq 0$, by Proposition 3.2, we see that $s_{2}\left(q_{1}, p_{1}, q_{2}, p_{2}\right)$ is a rational solution of $A_{5}^{(2)}\left(\alpha_{2}, \alpha_{2},-\alpha_{2}, \alpha_{3}+2 \alpha_{2}\right)$ such that all of $s_{0}\left(q_{1}, p_{1}, q_{2}, p_{2}\right)$ are holomorphic at $t=0$ and $a_{0,0} \neq 0, c_{0,0} \neq 0$. Based on the above discussion, considering that $\alpha_{0}+\alpha_{1}+2 \alpha_{2}+\alpha_{3}=1 / 2$, we can obtain the necessary condition.

The remaining case is that $\alpha_{0}=\alpha_{1}=\alpha_{2}=0, \alpha_{3}=1 / 2$. We prove that for $A_{5}^{(2)}(0,0,0,1 / 2)$, there exists no rational solution such that $q_{1}, p_{1}, q_{2}, p_{2}$ are all holomorphic at $t=0$ and $a_{0,0} \neq 0, c_{0,0} \neq 0$. If there exists such a rational solution, by Proposition 3.2, we find that $b_{0,0}=d_{0,0}=0$. Then, $s_{3}\left(q_{1}, p_{1}, q_{2}, p_{2}\right)$ is a rational solution of $A_{5}^{(2)}(0,0,1 / 2,-1 / 2)$ such that all of $s_{3}\left(q_{1}, p_{1}, q_{2}, p_{2}\right)$ are holomorphic at $t=0$ and $a_{0,0}=c_{0,0}=0$. Therefore, it follows from Proposition 6.2 that $-2 \cdot 0+(-1 / 2) \in \mathbb{Z}$, which is impossible. 


\subsection{The case where $p_{1}$ has a pole at $t=0$}

Proposition 6.5. Suppose that for $A_{5}^{(2)}\left(\alpha_{j}\right)_{0 \leq j \leq 3}$, there exists a rational solution such that $p_{1}$ has a pole at $t=0$ and $q_{1}, q_{2}, p_{2}$ are all holomorphic at $t=0$. Then, $-2 \alpha_{0}+\alpha_{3} \in \mathbb{Z}$, $\alpha_{3}-1 / 2 \in \mathbb{Z}$.

Proof. The proposition follows from Propositions 2.12, 3.3 and 4.9.

By $s_{1} s_{2}$, we can prove the following corollary.

Corollary 6.1. Suppose that for $A_{5}^{(2)}\left(\alpha_{j}\right)_{0 \leq j \leq 3}$, there exists a rational solution such that $p_{1}$ has a pole at $t=0$ and $q_{1}, q_{2}, p_{2}$ are all holomorphic at $t=0$. Then, by some Bäcklund transformations, the parameters can be transformed so that $-2 \alpha_{0}+\alpha_{3} \in \mathbb{Z},-2 \alpha_{1}+\alpha_{3} \in \mathbb{Z}$.

\subsection{The case where $p_{2}$ has a pole at $t=0$}

Proposition 6.6. Suppose that for $A_{5}^{(2)}\left(\alpha_{j}\right)_{0 \leq j \leq 3}$, there exists a rational solution such that $p_{2}$ has a pole at $t=0$ and $q_{1}, p_{1}, q_{2}$ are all holomorphic at $t=0$. Then, $-2 \alpha_{1}+\alpha_{3} \in \mathbb{Z}$, $\alpha_{3}-1 / 2 \in \mathbb{Z}$.

Proof. The proposition follows from Propositions 2.12, 3.4 and 4.9.

By $s_{0} s_{2}$, we can prove the following corollary.

Corollary 6.2. Suppose that for $A_{5}^{(2)}\left(\alpha_{j}\right)_{0 \leq j \leq 3}$, there exists a rational solution such that $p_{2}$ has a pole at $t=0$ and $q_{1}, p_{1}, q_{2}$ are all holomorphic at $t=0$. Then, by some Bäcklund transformations, the parameters can be transformed so that $-2 \alpha_{0}+\alpha_{3} \in \mathbb{Z},-2 \alpha_{1}+\alpha_{3} \in \mathbb{Z}$.

\subsection{Summary}

Proposition 6.7. Suppose that for $A_{5}^{(2)}\left(\alpha_{j}\right)_{0 \leq j \leq 3}$, there exists a rational solution. Then, one of the following occurs:

(1) $-2 \alpha_{0}+\alpha_{3} \in \mathbb{Z}, \quad-2 \alpha_{1}+\alpha_{3} \in \mathbb{Z}$,

(2) $-2 \alpha_{0}+\alpha_{3} \in \mathbb{Z}, \quad 2 \alpha_{1}+\alpha_{3} \in \mathbb{Z}$,

(3) $2 \alpha_{0}+\alpha_{3} \in \mathbb{Z}, \quad-2 \alpha_{1}+\alpha_{3} \in \mathbb{Z}$,

(4) $2 \alpha_{0}+\alpha_{3} \in \mathbb{Z}, \quad 2 \alpha_{1}+\alpha_{3} \in \mathbb{Z}$,

(5) $-2 \alpha_{0}+\alpha_{3} \in \mathbb{Z}, \quad \alpha_{3}-1 / 2 \in \mathbb{Z}$,

(6) $-2 \alpha_{1}+\alpha_{3} \in \mathbb{Z}, \quad \alpha_{3}-1 / 2 \in \mathbb{Z}$.

Corollary 6.3. Suppose that for $A_{5}^{(2)}\left(\alpha_{j}\right)_{0 \leq j \leq 3}$, there exists a rational solution. Then, by some Bäcklund transformations, the parameters can be transformed so that $-2 \alpha_{0}+\alpha_{3} \in \mathbb{Z}$, $-2 \alpha_{1}+\alpha_{3} \in \mathbb{Z}$.

\section{Necessary condition ... (2)}

\subsection{Shift operators}

In order to transform the parameters to the standard form, let us construct shift operators. 
Proposition 7.1. Let the shift operators $T_{0}, T_{1}, T_{2}$ be defined by

$$
T_{0}=\pi s_{2} s_{3} s_{2} s_{1} s_{0}, \quad T_{1}=s_{0} T_{0} s_{0}, \quad T_{2}=s_{2} T_{0} s_{2},
$$

respectively. Then,

$$
\begin{aligned}
& T_{0}\left(\alpha_{0}, \alpha_{1}, \alpha_{2}, \alpha_{3}\right)=\left(\alpha_{0}+1 / 2, \alpha_{1}+1 / 2, \alpha_{2}-1 / 2, \alpha_{3}\right), \\
& T_{1}\left(\alpha_{0}, \alpha_{1}, \alpha_{2}, \alpha_{3}\right)=\left(\alpha_{0}-1 / 2, \alpha_{1}+1 / 2, \alpha_{2}, \alpha_{3}\right), \\
& T_{2}\left(\alpha_{0}, \alpha_{1}, \alpha_{2}, \alpha_{3}\right)=\left(\alpha_{0}, \alpha_{1}, \alpha_{2}+1 / 2, \alpha_{3}-1\right),
\end{aligned}
$$

respectively.

\subsection{The properties of Bäcklund transformations}

\section{Proposition 7.2.}

(1) If $p_{1} \equiv 0$ for $A_{5}^{(2)}\left(\alpha_{j}\right)_{0 \leq j \leq 3}$, then $\alpha_{0}=0$.

(2) If $p_{2} \equiv 0$ for $A_{5}^{(2)}\left(\alpha_{j}\right)_{0 \leq j \leq 3}$, then $\alpha_{1}=0$.

(3) If $q_{1} q_{2}+t \equiv 0$ for $A_{5}^{(2)}\left(\alpha_{j}\right)_{0 \leq j \leq 3}$, then $\alpha_{2}=0$.

(4) If $p_{1}+p_{2}-1 \equiv 0$ for $A_{5}^{(2)}\left(\alpha_{j}\right)_{0 \leq j \leq 3}$, then $\alpha_{3}=0$.

By this proposition, we can consider $s_{0}$ as the identical transformation, if $p_{0} \equiv 0$. In the same way, we consider each of $s_{1}, s_{2}, s_{3}$ as the identical transformation, if $p_{2} \equiv 0$, or if $q_{1} q_{2}+t \equiv 0$, or if $p_{1}+p_{2}-1 \equiv 0$, respectively.

\subsection{Reduction of the parameters to the standard form}

By Corollary 6.3, using $T_{0}$, we can transform the parameters to $\left(\alpha_{0}, \alpha_{1}, \alpha_{2}, \alpha_{3}\right)=\left(\alpha_{3} / 2, \alpha_{3} / 2\right.$, $\left.\alpha_{2}, \alpha_{3}\right)$.

Proposition 7.3. Suppose that for $A_{5}^{(2)}\left(\alpha_{j}\right)_{0 \leq j \leq 3}$, there exists a rational solution. Then, by some Bäcklund transformations, the parameters can be transformed so that $-2 \alpha_{0}+\alpha_{3}=0$, $-2 \alpha_{1}+\alpha_{3}=0$.

\section{Classification of rational solutions}

\subsection{Rational solution of $A_{5}^{(2)}\left(\alpha_{3} / 2, \alpha_{3} / 2, \alpha_{2}, \alpha_{3}\right)$}

Proposition 8.1. For $A_{5}^{(2)}\left(\alpha_{3} / 2, \alpha_{3} / 2, \alpha_{2}, \alpha_{3}\right)$, there exists a rational solution and $\left(q_{1}, p_{1}, q_{2}\right.$, $\left.p_{2}\right)=(0,1 / 4,0,1 / 4)$. Moreover, it is unique.

Proof. The proposition follows from the direct calculation and Proposition 2.2.

\subsection{Proof of main theorem}

Let us prove our main theorem.

Proof. Suppose that for $A_{5}^{(2)}\left(\alpha_{j}\right)_{0 \leq j \leq 3}$, there exists a rational solution. Then, from Proposition 6.7, we find that the parameters satisfy one of the conditions in the theorem. Moreover, from Proposition 7.3, we see that the parameters can be transformed so that $-2 \alpha_{0}+\alpha_{3}=$ $-2 \alpha_{1}+\alpha_{3}=0$.

From Proposition 8.1, it follows that for $A_{5}^{(2)}\left(\alpha_{3} / 2, \alpha_{3} / 2, \alpha_{2}, \alpha_{3}\right)$, there exists a unique rational solution such that $\left(q_{1}, p_{1}, q_{2}, p_{2}\right)=(0,1 / 4,0,1 / 4)$, which proves the main theorem. 


\section{A Examples of rational solutions}

In this appendix, we give examples of rational solutions of $A_{5}^{(2)}\left(\alpha_{j}\right)_{0 \leq j \leq 3}$. For the purpose, we use the shift operators, $T_{0}, T_{1}, T_{2}$, and the seed rational solution,

$$
\left(q_{1}, p_{1}, q_{2}, p_{2}\right)=(0,1 / 4,0,1 / 4) \quad \text { for } \quad A_{5}^{(2)}\left(\alpha_{3} / 2, \alpha_{3} / 2, \alpha_{2}, \alpha_{3}\right) .
$$

Then, we obtain the following examples of rational solutions: for $A_{5}^{(2)}\left(\alpha_{3} / 2+1 / 2, \alpha_{3} / 2+1 / 2, \alpha_{2}-1 / 2, \alpha_{3}\right)$,

$$
\left(q_{1}, p_{1}, q_{2}, p_{2}\right)=\left(-1, \frac{1}{4}+\frac{\alpha_{3}}{2\left(t+4 \alpha_{3}^{2}\right)}-\frac{4 \alpha_{3}+1}{4(t+1)},-1, \frac{1}{4}-\frac{\alpha_{3}}{2\left(t+4 \alpha_{3}^{2}\right)}+\frac{4 \alpha_{3}+1}{4(t+1)},\right)
$$

for $A_{5}^{(2)}\left(\alpha_{3} / 2-1 / 2, \alpha_{3} / 2+1 / 2, \alpha_{2}, \alpha_{3}\right)$,

$$
\begin{aligned}
& \begin{array}{l}
q_{1}=2 \alpha_{3}-\frac{1}{1+\frac{\alpha_{3}\left(1-2 \alpha_{3}\right)}{t}}+\frac{-2 \alpha_{3}}{1+\frac{2 \alpha_{3}+1}{-t+\alpha_{3}\left(2 \alpha_{3}+1\right.}} \\
p_{1}=\frac{1}{4}+\frac{1}{-4 t+4 \alpha_{3}\left(2 \alpha_{3}+1\right)-\frac{\alpha_{3}\left(2 \alpha_{3}-1\right)}{t}},
\end{array} \\
& q_{2}=-\frac{1}{1-\frac{\alpha_{3}\left(2 \alpha_{3}-1\right)}{t}}, \\
& p_{2}=\frac{1}{4}+\frac{\alpha_{3}\left(2 \alpha_{3}-1\right)}{t}-\frac{2 \alpha_{3}+1}{\frac{-4}{1+\frac{\alpha_{3}\left(1-2 \alpha_{3}\right)}{t}}+\frac{4 t}{2 \alpha_{3}-\frac{1}{1+\frac{\alpha_{3}\left(1-2 \alpha_{3}\right)}{t}}} ;}
\end{aligned}
$$

for $A_{5}^{(2)}\left(\alpha_{3} / 2, \alpha_{3} / 2, \alpha_{2}+1 / 2, \alpha_{3}-1\right)$,

$$
\begin{aligned}
\left(q_{1}, p_{1}, q_{2}, p_{2}\right)= & \left(-1, \frac{1}{4}-\frac{2 \alpha_{3}-1}{4\left\{t+\left(2 \alpha_{3}-1\right)^{2}\right\}}+\frac{4 \alpha_{3}-3}{2(t+1)},-1,\right. \\
& \left.\frac{1}{4}+\frac{2 \alpha_{3}-1}{4\left\{t+\left(2 \alpha_{3}-1\right)^{2}\right\}}-\frac{4 \alpha_{3}-3}{2(t+1)}\right) .
\end{aligned}
$$

\section{Acknowledgments}

The author wishes to express his sincere thanks to Professor Yousuke Ohyama. In addition, he is also indebted the referees for their useful comments. 


\section{References}

[1] Airault H., Rational solutions of Painlevé equation, Stud. Appl. Math. 61 (1979), 31-53.

[2] Bassom A.P., Clarkson P.A., Hicks A.C., Bäcklund transformations and solution hierarchies for the fourth Painlevé equation, Stud. Appl. Math. 95 (1995), 1-71.

[3] Clarkson P.A., The third Painlevé equation and associated special polynomial, J. Phys. A: Math. Gen. 36 (2003), 9507-9532.

[4] Gambier B., Sur les équations différentielles du second ordre et du premier degré dont l'intégrale générale est a points critique fixes, Acta Math. 33 (1910), 1-55.

[5] Gromak V.I., Algebraic solutions of the third Painlevé equation, Dokl. Akad. Nauk BSSR 23 (1979), 499-502 (in Russian).

[6] Gromak V.I., Reducibility of the Painlevé equations, Differ. Equ. 20 (1983), 1191-1198.

[7] Hone A.N.W., Coupled Painlevé systems and quartic potentials, J. Phys. A: Math. Gen. 34 (2001), 22352246.

[8] Kitaev A.V., Law C.K., McLeod J.B., Rational solutions of the fifth Painlevé equation, Differential Integral Equations 7 (1994), 967-1000.

[9] Matsuda K., Rational solutions of the $A_{4}$ Painlevé equation, Proc. Japan Acad. Ser. A Math. Sci. 81 (2005), no. $5,85-88$.

[10] Matsuda K., Rational solutions of the $A_{5}^{(1)}$ Painlevé equation, arXiv:0708.2960.

[11] Mazzoco M., Rational solutions of the Painlevé VI equation, J. Phys. A: Math. Gen. 34 (2001), 2281-2294, nlin.SI/0007036.

[12] Mazzoco M., Mo M.Y., The Hamiltonian structure of the second Painlevé hierarchy, Nonlinearity 20 (2007), 2845-2882, nlin.SI/0610066.

[13] Milne A.E., Clarkson P.A., Bassom A.P., Bäcklund transformations and solution hierarchies for the third Painlevé equation, Stud. Appl. Math. 98 (1997), 139-194.

[14] Murata Y., Rational solutions of the second and the fourth Painlevé equations, Funkcial. Ekvac. 28 (1985), $1-32$.

[15] Murata Y., Classical solutions of the third Painlevé equation, Nagoya Math. J. 139 (1995), 37-65.

[16] Noumi M., Yamada Y., Higher order Painlevé equations of type $A_{l}^{(1)}$, Funkcial. Ekvac. 41 (1998), 483-503, math.QA/9808003.

[17] Okamoto K., Studies on the Painlevé equations. III. Second and fourth Painlevé equations, $P_{\mathrm{II}}$ and $P_{\mathrm{IV}}$, Math. Ann. 275 (1986), 221-255.

[18] Okamoto K., Studies on the Painlevé equations. I. Sixth Painlevé equation PVI, Ann. Mat. Pure Appl. (4) 146 (1987), 337-338.

[19] Okamoto K., Studies on the Painlevé equations. II. Fifth Painlevé equation P. Japan. J. Math. (N.S.) 13 (1987), 47-76.

[20] Okamoto K., Studies on the Painlevé equations. IV. Third Painlevé equation PIII, Funkcial. Ekvac. 30 (1987), 305-332.

[21] Painlevé P., Sur les équations différentielles du second ordre et d'ordre supérieur dont l'intégrale générale est uniforme, Acta Math. 25 (1902), 1-85.

[22] Sasano Y., Higher order Painlevé equations of type $D_{l}^{(1)}$, RIMS Kokyuroku 1473 (2006), no. 1, $43-163$.

[23] Sasano Y., Symmetries in the system of type $A_{5}^{(2)}$, arXiv:0704.2327.

[24] Vorob'ev A.P., On rational solutions of the second Painlevé equation, Differ. Equ. 1 (1965), 58-59.

[25] Yablonskii A.I., On rational solutions of the second Painlevé equation, Vesti AN BSSR, Ser. Fiz.-Tech. Nauk (1959), no. 3, 30-35 (in Russian).

[26] Yuang W., Li Y., Rational solutions of Painlevé equations, Canad. J. Math. 54 (2002), 648-670. 